\title{
CHARACTERIZATION OF THE SPEED OF A TWO-PHASE INTERFACE IN A POROUS MEDIUM
}

ADIL ABBASSI AND GAWTUM NAMAH

Received 8 November 2004 and in revised form 15 February 2005

A typical situation of oil reservoir simulation is considered in a porous medium where the resident oil is displaced by water injection. An explicit expression of the speed of the oilwater interface is given in a pseudo-2D case via the resolution of an auxiliary Riemann problem. The explicit 2D solution is then corroborated with numerical simulations by solving the transport equation with a generalized scheme of Harten type.

\section{Introduction}

Let $\Omega$ be a rectangular domain in $\mathbb{R}^{2}$ and let $\partial \Omega$ be its boundary. In this paper, we will be interested in the resolution of a system of type

$$
(P) \begin{cases}\varepsilon \frac{\partial u}{\partial t}+\operatorname{div}(V f(u))=0 & \text { in } \Omega \times(0,+\infty), \\ \operatorname{div} V=0 & \text { in } \Omega\end{cases}
$$

with

$$
V=-m(u) \nabla p
$$

subject to the initial condition

$$
u(0, x)=u_{0}(x), \quad x \in \Omega,
$$

and the following boundary condition for the variable $u$ :

$$
u(t, x)=1 \quad \text { on } \Gamma_{\text {in }}
$$

together with the conditions for $p$ :

$$
p=1 \quad \text { on } \Gamma_{\text {out }}, \quad V \cdot n=-m(u) \frac{\partial p}{\partial n}=g \quad \text { on } \partial \Omega \backslash \Gamma_{\text {out }}
$$

and where $\Gamma_{\text {in }}$ and $\Gamma_{\text {out }}$ are as defined below. 


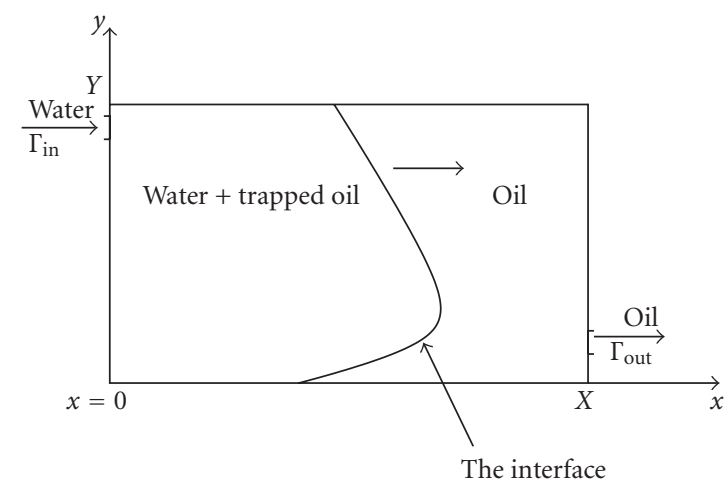

Figure 1.1. Water-oil reservoir simulation in porous media.

Equations (1.1)-(1.3) represent a simplified model for an incompressible flow in some porous medium, analogous to the Buckley-Leverett model used in oil reservoir simulations [9]. In this context, the system represents a water-oil displacement model, that is, oil trapped in $\Omega$ is pushed towards $\Gamma_{\text {out }}$ by injection of water at $\Gamma_{\text {in }}$, see Figure 1.1. In our case, the variable $u$ represents the saturation of the water phase, the vector $V$ is the total flux density of the two fluids, and $p$ is the pressure. See next paragraph for the derivation of the model.

The functions $f$ and $m$ are nonlinear functions of $u$. A typical representation of the fractional flow function $f$, which characterizes the relative permeability of the fluids, is given for some $\alpha>0$ by

$$
f(u)=\frac{\lambda u^{\alpha}}{\lambda u^{\alpha}+(1-u)^{\alpha}}
$$

(see Figure 1.2). In the above formula, $\lambda$ is the viscosity ratio of the two fluids $\left(\lambda=\mu_{o} / \mu_{w}\right)$. Note that such an $f$, which is obtained by considering a relative permeability of the polynomial form $u^{\alpha}$, is an increasing function but which may change concavity. As concerning the function $m$, it will be supposed to be positive, bounded, that is, there exist $m_{0}$ and $m_{1}$ such that

$$
0<m_{0} \leq m(u) \leq m_{1}<\infty
$$

for $u \in[0,1]$. More precisely, $m$, which represents the total mobility of the two fluids, is given by

$$
m(u)=\frac{u^{\alpha}}{\mu_{w}}+\frac{(1-u)^{\alpha}}{\mu_{o}}=\frac{\lambda u^{\alpha}+(1-u)^{\alpha}}{\lambda \mu_{w}}
$$

Multiphase flows in porous media are inherently nonlinear and often numerical simulations happen to be the only practical way to understand their qualitative behaviour. 


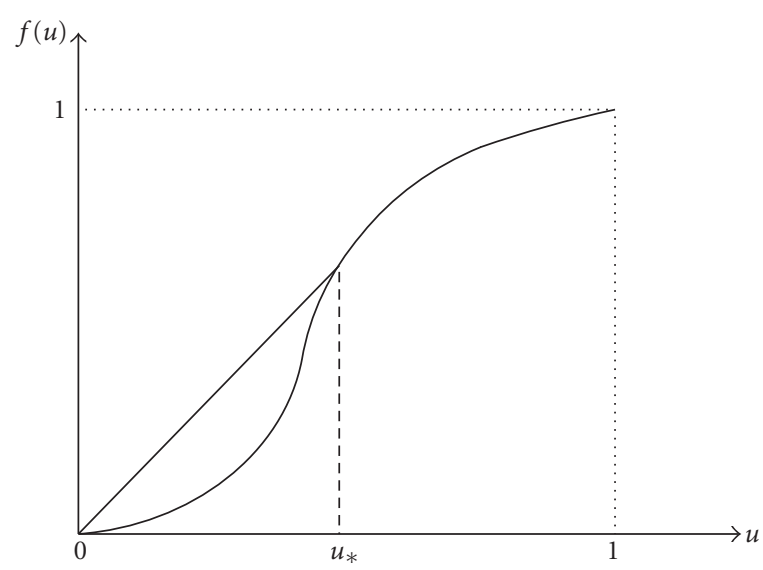

Figure 1.2. Typical profile of the fractional flow function $f$.

Even for simplified systems analogous to (1.1)-(1.2), usually named the Buckley-Leverett model, there is no general result concerning the existence and uniqueness of a global solution of such a system, see, for example, the book of Gagneux et al. [14]. This hyperbolicelliptic-type model results by neglecting gravity and capillary forces in the generalized Darcy's law which is usually used for the expression of the velocity in the case of two immiscible fluids sharing a porous medium (cf. $[2,5,10,14])$. This leads to the expression (1.2) in our case. The consideration of the capillary forces would result in a parabolicelliptic version studied by various authors, see, for example, [13, 14]. As for the hyperbolic case, results which exist concern essentially treatment of particular boundary conditions. For example, in [14], the mobility $m$ is taken to be a constant which comes to solving $-\Delta p=0$ for the elliptic part. This together with convenient boundary conditions of Neumann type on $\Gamma_{\text {in }}$ and Robin-type condition on $\Gamma_{\text {out }}$ ensures a unique solution in $C\left([0, T] ; L^{1}(\Omega)\right)$ for all $T>0$. In their paper, Schroll and Tveito [27] are interested in the local existence and stability of solutions of such a system. With Neumann conditions on the whole boundary and appropriate regularity hypotheses, they indeed obtain a classical but local in time solution.

In this paper, we propose to study the well-posedness of the system (1.1)-(1.5) and to construct a weak entropy solution in the pseudo-2D case (i.e., when $\Gamma_{\text {in }}$ and $\Gamma_{\text {out }}$ are the lateral sides of the rectangular domain) via the resolution of an auxiliary $1 \mathrm{D}$ problem of Riemann type.

We start by describing the simplications called for in the physical problem which led to our model by departing from the theory of mixtures, see, for example, [2, 25]. Then in Section 3 we define an entropy solution of (1.1)-(1.5), and we verify that the problem is well-posed in the sense of Bardos-LeRoux-Nédélec (BLN) [6]. In Section 4, we solve the corresponding one-dimensional problem which in turn allows us to give an entropy solution in the pseudo-2D case. In the last section, some numerical results are presented, to corroborate the theoretical ones, by using a scheme of Harten type. 


\section{Derivation of the model}

2.1. The theory of mixtures. As we said in the introduction, the system (1.1)-(1.5) represents a simplified water-oil displacement model used in oil reservoir simulations for enhanced oil recovery. The latter consists in injecting water at a well $\left(\Gamma_{\text {in }}\right)$ in order to push the oil trapped in the porous rock $(\Omega)$ towards the production well $\left(\Gamma_{\text {out }}\right)$.

A complete theoretical model must therefore be able to take into account the various physical characteristics and any eventual interactions of the three phases in presence: the two fluids oil $(o)$ and water $(w)$ and the solid rock $(r)$. The so-called theory of mixtures provides such a framework as it presents a systematic methodology to derive equations of problems such as flow through a porous medium. For an overview of models concerning multiphase flows, we refer to the book on Mechanics of Mixture by Rajagopal and Tao [25], and among other papers those of Atkin and Craine [4], Bowen [8], Truesdell [28], and Allen [2].

Let $p$ be the index which denotes the phase (in our case $p=o, w$, or $r$ ) and let $\phi_{p}, \rho_{p}$, and $v_{p}$ represent, respectively, the volume fraction, the mass density, and the velocity of the phase $p$. Then the porosity of the medium is given by

$$
\phi=1-\phi_{r}\left(=\phi_{w}+\phi_{o}\right)
$$

The saturation $S_{p}$ of the fluid phase $p$ is then defined by

$$
S_{p}=\frac{\phi_{p}}{\phi}, \quad p=o, w
$$

subject of course to the constraint

$$
S_{w}+S_{o}=1
$$

The governing equations of an isothermal flow will be obtained by writing the mass and momentum balance equations for the three phases. As in our case there is supposed to be no interphase mass transfer and, in particular, the rock is chemically inert, the mass conservation for any phase $p$ will read

$$
\frac{\partial\left(\phi_{p} \rho_{p}\right)}{\partial t}+\nabla \cdot\left(\phi_{p} \rho_{p} v_{p}\right)=0, \quad p=o, w, r .
$$

The velocity field of the rock can be reasonably taken as zero (up to fixing a coordinate system in which $v_{r}=0$ ). In particular, we neglect any deformation which could have been caused by the water injection. As concerning the velocity fields of the fluids, they are obtained via the momentum balance equations which for Newtonian fluids are given by

$$
\phi_{p} \rho_{p} \frac{D v_{p}}{D t}=-\nabla P_{p}+\rho_{p} g \nabla z+m_{p}, \quad p=o, w
$$

where $P_{p}$ is the mechanical pressure in the fluid $p, g$ the gravitational acceleration, $z$ some depth, and $m_{p}$ the momentum exchange from other phases. This latter quantity is 
commonly assumed to take the form

$$
\Lambda_{p} m_{p}=\phi_{p}\left(v_{r}-v_{\alpha}\right)
$$

where $\Lambda_{p}$ is the mobility tensor of the phase $p$. If we further assume that the inertial effects in the fluids are negligible (i.e., $\left.D v_{p} / D t=0\right),(2.5)$ yields the so-called Darcy's law

$$
\phi_{p} v_{p}=\Lambda_{p}\left(-\nabla P_{p}+\rho_{p} g \nabla z\right) .
$$

Constitutive laws for mobility are largely phenomenological, the most common versions having the form

$$
\Lambda_{p}=\frac{\mathbf{k} K_{r p}}{\mu_{p}},
$$

where $\mu_{p}$ is the dynamic viscosity of fluid phase $p, \mathbf{k}$ is the permeability tensor, and the relative permeability $K_{r p}$ is a coefficient describing the effects of the other fluid in obstructing the flow of fluid $p$. For a two-fluid system with no interphase mass transfer as in our case, $K_{r p}$ is typically a function of the saturation $S_{p}$.

2.2. The Buckley-Leverett model. The Buckley-Leverett model [9] is a simplified model of two phase flows in porous media but which is of particular relevance in the petroleum industry for enhanced oil recovery. This model is based on some basic assumptions, namely, the incompressibility of the solid and the fluids and the fact that the effects of capillary pressure gradients on the flow fields are negligible as compared to the pressure gradients applied through pumping.

The incompressibility assumption implies that $\phi$ is constant in time and that the fluids densities are constant in space and time. One can then simplify the continuity equation (2.4) by $\rho_{p}$ and it becomes

$$
\frac{\partial \phi_{p}}{\partial t}+\nabla \cdot\left(\phi_{p} v_{p}\right)=0, \quad p=o, w
$$

If we introduce the flux function $V_{p}$ defined by

$$
V_{p}=\phi_{p} v_{p}
$$

and use (2.2), we get

$$
\phi \frac{\partial S_{p}}{\partial t}+\nabla \cdot V_{p}=0, \quad p=o, w
$$

By summing the equations for the two fluids and using the fact that $S_{o}+S_{w}=1$, we then have the system

$$
\begin{gathered}
\phi \frac{\partial\left(S_{w}\right)}{\partial t}+\operatorname{div} V_{w}=0, \\
\operatorname{div} V_{T}=0
\end{gathered}
$$


with $V_{T}=V_{o}+V_{w}$, the total flux given by, due to (2.7),

$$
V_{T}=\Lambda_{w}\left(-\nabla P_{w}+\rho_{w} g \nabla z\right)+\Lambda_{o}\left(-\nabla P_{o}+\rho_{o} g \nabla z\right) .
$$

In practice, the interface tension, which occurs due to the difference of pressures of the two fluids, leads to capillary effects. In our two-phase system, there will be a single capillary pressure $P_{c}$ given by

$$
P_{c}=P_{o}-P_{w}
$$

Now we invoke the assumption of the Buckley-Leverett model corresponding to the fact that capillarity has negligible effects on the flow field-wide, that is, $\operatorname{grad} P_{c} \simeq 0$ so that

$$
\nabla P_{w}=\nabla P_{o}(=\nabla P)
$$

Finally assuming that gravity effects are absent as in the $1 \mathrm{D}$ case, the expression of $V_{T}$ collapses to

$$
V_{T}=-\left(\Lambda_{w}+\Lambda_{o}\right) \nabla P
$$

Now define the fractional flow of water, $f=f\left(S_{w}\right)$, by

$$
f=\frac{\Lambda_{w}}{\Lambda_{o}+\Lambda_{w}}
$$

By noting that $V_{w}=V_{T} f$, we thus end up with the hyperbolic-type equation

$$
\phi \frac{\partial S_{w}}{\partial t}+\operatorname{div}\left(V_{T} f\right)=0
$$

coupled to

$$
\operatorname{div} V_{T}=0
$$

with $V_{T}$ given by (2.16). If we now define the total mobility by $m$, that is,

$$
m=\Lambda_{w}+\Lambda_{o}
$$

then one sees that it suffices to replace in the above equations the porosity $\phi$ by $\varepsilon$, the unknown $S_{w}$ by $u$, and the total flux function $V_{T}$ by $V$ to get (1.1)-(1.2).

The fractional flow $f$, which is clearly nonlinear through its dependence on the unknown $S_{w}$, has typically an "S-shaped" profile such as the one depicted in (1.6). As concerning the total mobility function $m$, due to (2.8), it is given by

$$
m=\mathbf{k}\left(\frac{K_{r o}}{\mu_{o}}+\frac{K_{r w}}{\mu_{w}}\right) .
$$

The expression (1.8) considered in this paper is thus obtained by considering an identity permeability tensor $\mathbf{k}$ (which comes to assuming that the rock is homogeneous without any particular isotropy) and a polynomial form of the relative permeability, $K_{r w}=S_{w}^{\alpha}$. 
Let us finally remark that not neglecting the capillary forces would lead to completely different models on a mathematical basis. Usually one ends up with quasilinear parabolic degenerate equations instead of hyperbolic ones - see, for example, Chavent and Jaffre [10] where a fictitious global pressure is introduced as a new unknown to weaken the coupling between $S$ and $P$.

\section{Definition and well-posedness}

Let us first say a few words on the boundary conditions. Notice that the boundary condition for $u$ is given only at $\Gamma_{\text {in }}$. In fact, as we will see further, this is sufficient for the well-posedness of the problem in the sense of BLN [6]. As concerning the boundary conditions on $p$, we have $p=1$ at $\Gamma_{\text {out }}$ corresponding to an atmospheric pressure, which is physically meaningful. But without loss of generality for the analysis, we will consider from now on $p=0$ at $\Gamma_{\text {out }}$. The condition on the rest of the boundary is

$$
-m(u) \frac{\partial p}{\partial n}=g \quad \text { on } \partial \Omega \backslash \Gamma_{\text {out }}
$$

$n$ being the outward unit normal. As for $g$, it will be taken as follows:

$$
g= \begin{cases}-q & \text { on } \Gamma_{\text {in }}, \\ 0 & \text { elsewhere }\end{cases}
$$

with some positive constant $q$. In this context, $q$ represents the injection speed of the water at $\Gamma_{\text {in }}$, the rest of the boundary being impermeable except of course for $\Gamma_{\text {out }}$. Let us note that such a boundary condition is indeed not a regular one ( $g$ is not continuous) and the latter could have been regularized but we will not do so as regularity results are not our aim here. Instead we prefer a context which physically makes sense and deals with the weak formulation below.

Define $Q=\Omega \times] 0, T$ [ for some time $T>0$ and let $B V$ be the space of functions which have bounded variations. Consider then the following problem for a given $u$ in $L^{\infty} \cap$ $B V(Q)$ and $t \in] 0, T\left[\right.$ : find $p(\cdot, t) \in \mathscr{V}=\left\{v \in H^{1}(\Omega), v=0\right.$ on $\left.\Gamma_{\text {out }}\right\}$ such that for all $\varphi \in \mathscr{V}$

$$
\int_{\Omega} m(u(\cdot, t)) \nabla p \nabla \varphi d x d y=\int_{\Gamma_{\mathrm{in}}} q \varphi d s
$$

Of course, such a $p$ will depend on $t$ via the function $u(\cdot, t)$. Note that as $u \in B V(Q)$, $u(\cdot, t)$ can be defined for all $t>0$ in the sense of trace.

Proposition 3.1. Let $\operatorname{mes}\left(\Gamma_{\text {in }}\right)>0$ and mes $\left(\Gamma_{\text {out }}\right)>0$. Then for any given $u(\cdot, t) \in L^{\infty} \cap$ $B V(\Omega)$, the problem (3.3) admits a unique solution $p$ in $\mathscr{V}$. Moreover, this solution is positive a.e. in $\Omega$. 
648 A two-phase interface in a porous medium

Proof. Set

$$
a(p, \varphi)=\int_{\Omega} m(u) \nabla p \nabla \varphi d x d y
$$

Then denoting by $|\cdot|_{1, \Omega}$ (resp., $\|\cdot\|_{1, \Omega}$ ) the seminorm (resp., the norm) on $H^{1}(\Omega)$, we have, for $v \in \mathscr{V}$,

$$
a(v, v) \geq m_{0}|v|_{1, \Omega}^{2}
$$

and, as $m_{0}>0$, Poincaré's inequality which holds on $\mathscr{V}$ leads to

$$
a(v, v) \geq C_{1}\|v\|_{1, \Omega}^{2}
$$

We then conclude by Lax-Milgram for the existence and uniqueness of $p$.

Let then

$$
p=p^{+}-p^{-}
$$

where $p^{-}=\sup (-p, 0)$. By taking $\varphi=p^{-}$as the test function in the problem (3.3), we have

$$
\begin{aligned}
a\left(p, p^{-}\right) & =\int_{\Omega} m(u) \nabla p^{+} \nabla p^{-} d x d y-\int_{\Omega} m(u)\left|\nabla p^{-}\right| d x d y \\
& =-\int_{\Omega} m(u)\left|\nabla p^{-}\right| d x d y \leq 0
\end{aligned}
$$

On the other hand,

$$
a\left(p, p^{-}\right)=\int_{\Gamma_{\text {in }}} q p_{\Gamma_{\Gamma_{\text {in }}}}^{-} d y \geq 0
$$

Thus we have

$$
\int_{\Omega} m(u)\left|\nabla p^{-}\right| d x d y=0
$$

which leads to the fact that $p^{-}$is almost everywhere constant on $\Omega$. And, as $p_{\left.\right|_{\Gamma_{\text {out }}}}^{-}=0$, we finally have $p \geq 0$ a.e. on $\Omega$.

Note that, as $p$ can only be decreasing across $\Gamma_{\text {out }}$, the above proposition ensures that it suffices to impose a boundary condition for $u$ only on $\Gamma_{\text {in }}$ for the problem to be well-posed in the sense of BLN [6]. Indeed the correct way to prescribe the boundary conditions for the hyperbolic part in our case happens to be

$$
\min _{k \in(\gamma(u), 1)}\{\operatorname{sg}(\gamma(u)-1)[f(\gamma(u))-f(k)] \vec{V} \cdot \vec{n}\}=0 .
$$


As $f$ is increasing, (3.11) is equivalent to

$$
\min _{k \in(\gamma(u), 1)}\{|f(\gamma(u))-f(k)| \vec{V} \cdot \vec{n}\}=0
$$

This last equality will always hold if we impose $\gamma(u)=1$ on the part of the boundary where $\vec{V} \cdot \vec{n}<0$, that is, on $\Gamma_{\text {in }}$.

We now proceed by giving the definition of a solution of (1.1)-(1.5). For this sake and without loss of generality, we will shift to homogeneous Dirichlet boundary conditions for the hyperbolic part which is obtained by considering the unknown $(u-1)$ instead of $u$. Define then the function $h$ by

$$
h(z)=-\frac{1}{\varepsilon} m(z+1) f(z+1) .
$$

Definition 3.2. Given $u_{0} \in B V(\Omega) \cap L^{\infty}(\Omega)$, the pair $(u, p)$ will be called an entropy solution of (1.1)-(1.5) if

(i) $u \in B V(Q) \cap L^{\infty}(Q)$ satisfies the entropy inequality

$$
\left(P_{u}\right)\left\{\begin{array}{l}
\forall \phi \in D(\bar{\Omega} \times[0, T[), \phi \geq 0, \forall k \in \mathbb{R}, \\
\int_{Q}\left\{|u-k| \frac{\partial \phi}{\partial t}+\operatorname{sg}(u-k)\left[h(u) \nabla p-h(k) \nabla p_{k}\right] \nabla \phi\right\} d x d y d t \\
-\int_{Q} \operatorname{sg}(u-k) \operatorname{div}\left(h(k) \nabla p_{k}\right) \phi d x d y d t \\
+\int_{0}^{T} \int_{\Gamma}\left\{\operatorname{sg}(k)\left[h(\gamma(u)) \nabla p-h(k) \nabla p_{k}\right] \cdot \vec{n}\right\} \phi d \sigma d t \\
+\int_{\Omega}\left|u_{0}-k\right| \phi(\cdot, 0) d x d y \geq 0,
\end{array}\right.
$$

(ii) $p \in L^{\infty}(0, T$; $\mathscr{V})$ satisfies the weak formulation (3.3) for almost all $\left.t \in\right] 0, T[$.

In the above definition, $p_{k}$ is the unique solution of (3.3) when $u$ is replaced by the constant $k$ and $\gamma(u)$ represents the trace of $u$ in $L^{\infty}(\partial \Omega \times] 0, T[)$ and in $L^{\infty}(\Omega)$ for $t=0$. This trace is well defined for $B V$ functions (cf. LeRoux [20]). And finally sg is the usual sign function.

This definition is inspired by that of BLN [6] where it is given for a general flux function $f=f(x, u, t)$. In our case, the corresponding flux is $h(u) \nabla p$ and by taking the entropy pair $(U, F)$ as

$$
U(u)=|u-k|, \quad F(u)=\operatorname{sg}(u-k)\left(h(u) \nabla p-h(k) \nabla p_{k}\right),
$$


we recover the definition of [6] with $h(u) \nabla p$ replaced by $f(x, u, t)$. Also in the same context of a porous medium flow, a definition is given for such a function in [14] but where $m$ is taken as a constant. Consequently this simplication led to the decoupling of the problem $P_{u}$ from the problem (3.3).

\section{A 1D Riemann problem}

A first simplification in the $1 \mathrm{D}$ case is that the incompressibility condition $\operatorname{div} V=0 \mathrm{im}$ plies the independence of $V$ with respect to the space variable $x$ so that we have

$$
V(x, t)=V(0, t), \quad \forall t>0
$$

As the total velocity at $x=0$ is equal to $q$, we therefore end up with

$$
V(x, t)=q, \quad \forall x \geq 0, t \geq 0 .
$$

Let us then consider the scalar conservation equation

$$
u_{t}+\frac{q}{\varepsilon} f(u)_{x}=0, \quad x \in \mathbb{R}
$$

with an initial condition of Riemann type

$$
u(x, 0)= \begin{cases}1, & x<0 \\ 0, & x>0\end{cases}
$$

The pressure $p$ is then obtained by solving

$$
-m(u) \frac{\partial p}{\partial x}=q
$$

Let us point out that equations of type (4.3)-(4.4) can be completely solved by using hyperbolic techniques (cf., e.g., [15]). But before doing so, let us recall the following results.

Proposition 4.1. Equations (4.3)-(4.4) admit a unique entropy solution in $L^{\infty}(\mathbb{R} \times(0, T))$, for all $T>0$. This solution can only be discontinuous along lines of equations $x / t=$ constant. Moreover, $|u(\cdot, t)|_{L^{\infty}(\mathbb{R})} \leq 1$ a.e.

In fact, the resolution depends on the profile of the fractional flow $f$. In our case, we have the following.

Proposition 4.2. Let $\alpha>0$. Then $f$ which is given by (1.6), that is,

$$
f(u)=\frac{\lambda u^{\alpha}}{\lambda u^{\alpha}+(1-u)^{\alpha}},
$$

is either affine $(\alpha=1, \lambda=1)$, strictly convex $(\alpha=1, \lambda<1)$, strictly concave $(\alpha=1, \lambda>1)$, or admits at most one inflexion point in the interval $(0,1)$. 
The proof is omitted as it results from tedious but straightforward calculations. In fact, if $\alpha<1$, then $f$ changes concavity from concave to convex whereas for $\alpha>1, f$ is first convex before becoming concave as we go from $u=0$ to $u=1$.

Now let $\sum$ be a line of discontinuity (a shock). Denote by $u^{+}$(resp., $u^{-}$) the value of the solution on the right (resp., left) of $\sum$. Then the speed (c) of the shock is given by the Rankine-Hugoniot conditions:

$$
c=\frac{f\left(u^{+}\right)-f\left(u^{-}\right)}{u^{+}-u^{-}} .
$$

We then have the following.

Proposition 4.3 (Oleinik). $u$ is an entropy solution of (2.16)-(2.18) if and only if one of the following three conditions is verified.

(a) For all $k \in\left(u^{-}, u^{+}\right), c \geq\left(f\left(u^{+}\right)-f(k)\right) /\left(u^{+}-k\right)$.

(b) For all $k \in\left(u^{-}, u^{+}\right), c \leq\left(f\left(u^{-}\right)-f(k)\right) /\left(u^{-}-k\right)$.

(c) Let $\beta \in[0,1]$.

$$
\begin{aligned}
& \text { If } u^{+}>u^{-} \text {, then } f\left(\beta u^{-}+(1-\beta) u^{+}\right) \geq \beta f\left(u^{-}\right)+(1-\beta) f\left(u^{+}\right) \text {. } \\
& \text { If } u^{-}>u^{+} \text {, then } f\left(\beta u^{-}+(1-\beta) u^{+}\right) \leq \beta f\left(u^{-}\right)+(1-\beta) f\left(u^{+}\right) .
\end{aligned}
$$

Note that if $f$ is strictly convex, $u$ will be an entropy solution if and only if the shock is decreasing, that is, $u^{+}<u^{-}$. Now we solve for the different cases.

(i) $\alpha=1, \lambda \leq 1$. In that case, $f$ is affine or strictly convex. Note that the initial discontinuity is admissible and it propagates with speed $c$ (cf. (4.7)) given in our case by

$$
c=\frac{q}{\varepsilon} .
$$

The corresponding solution, at time $t>0$, is then given by

$$
u(x, t)= \begin{cases}1, & x<c t, \\ 0, & x>c t .\end{cases}
$$

(ii) $\alpha=1, \lambda>1$. The flow function $f$ being strictly concave, the initial discontinuity is not admissible, so that $u^{-}$and $u^{+}$will be connected by a rarefaction wave. The solution at some time $t>0$ is given by

$$
u(x, t)= \begin{cases}1, & \frac{x}{t} \leq f^{\prime}\left(u^{-}\right)=\frac{q}{\lambda \varepsilon}, \\ \left(f^{\prime}\right)^{-1}\left(\frac{x}{t}\right), & \frac{q}{\lambda \varepsilon} \leq \frac{x}{t} \leq \frac{\lambda q}{\varepsilon}, \\ 0, & \frac{x}{t} \geq f^{\prime}\left(u^{+}\right)=\frac{\lambda q}{\varepsilon} .\end{cases}
$$

(iii) $\alpha>1$. When $\alpha>1, f$ admits one inflexion point. We will therefore expect at most one shock. By considering the upper concave envelope of $f$, we define the point $u_{*}$ 


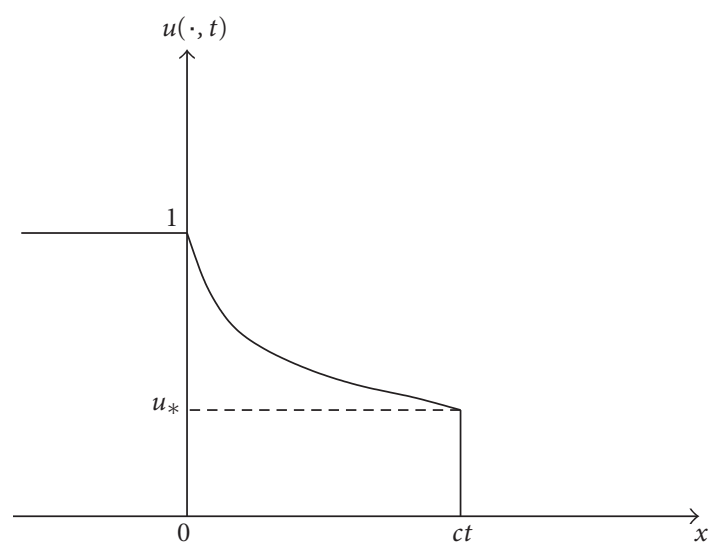

Figure 4.1. A rarefaction wave followed by the interface discontinuity.

(cf. Figure 1.2), given by

$$
f^{\prime}\left(u_{*}\right)=\frac{f\left(u_{*}\right)}{u_{*}}
$$

The saturation $u_{*}$, which corresponds to the point of change of concavity of the flow function $f$, is known as the Welge saturation in oil reservoir simulations. In the interval $\left(u_{*}, 1\right), f$ is strictly concave so that two states $u_{*}$ and 1 can be connected by a rarefaction wave

$$
u(x, t)=\left(f^{\prime}\right)^{-1}\left(\frac{x}{t}\right), \quad 0<x<\frac{q}{\varepsilon} f^{\prime}\left(u_{*}\right) t
$$

whereas the states $u_{*}$ and 0 will be connected by a shock wave (Proposition 4.3) which propagates with speed

$$
c=\frac{q}{\varepsilon} \frac{f\left(u_{*}\right)}{u_{*}} .
$$

We therefore end up with a rarefaction wave followed by a shock (cf. Figure 4.1).

(iv) $\alpha<1$. And finally the last case corresponds to a function $f$ which is concave then becomes convex. By again considering the upper concave envelope, we define $u_{*}$ which satisfies

$$
f^{\prime}\left(u_{*}\right)=\frac{1-f\left(u_{*}\right)}{1-u_{*}}
$$

Then the states 1 and $u_{*}$ can be connected by a shock (Proposition 4.3) at $x=f^{\prime}\left(u_{*}\right) t$ 
followed by a rarefaction wave given by

$$
u(x, t)=\left(f^{\prime}\right)^{-1}\left(\frac{x}{t}\right), \quad f^{\prime}\left(u_{*}\right) t \leq x \leq f^{\prime}(0) t
$$

This completes the resolution in the $1 \mathrm{D}$ case.

Note that the value $\alpha>1$ seems to be the most realistic one. In that case, we end up with the following result concerning the speed of the oil-water interface.

THeOREM 4.4. Let the flow function $f$ be given by

$$
f(u)=\frac{\lambda u^{\alpha}}{\lambda u^{\alpha}+(1-u)^{\alpha}}
$$

with $\alpha>1$. Define $u_{*} \in[0,1]$ which solves

$$
\lambda u^{\alpha}+(1-u)^{\alpha}-\alpha(1-u)^{\alpha-1}=0 .
$$

Then the water saturation is given by the profile of Figure 4.1 and the speed of the oil-water interface is a constant given by

$$
c=\frac{q}{\varepsilon} \frac{\lambda u_{*}^{\alpha-1}}{\lambda u_{*}^{\alpha}+\left(1-u_{*}\right)^{\alpha}} .
$$

For example, when $\alpha=2$ (a value typically considered for models with a nonlinear permeability), the speed is given by

$$
c=\frac{q}{\varepsilon} \frac{1+\sqrt{1+\lambda}}{2}
$$

In fact, we use the previous resolution for the case $\alpha>1$ where $u_{*}$, given by (4.11), can be explicitly computed and is found to verify (4.17). The discontinuity in our case happens to be the oil-water interface so that the latter propagates with the speed $\mathrm{c}$ given by the Rankine-Hugoniot condition and the proof is done.

The profile of $p$. Recall that the pressure $p$ is given for any time $t=t^{*}$ by

$$
\frac{\partial p}{\partial x}\left(x, t^{*}\right)=\frac{-q}{m\left(u\left(x, t^{*}\right)\right)}, \quad x>0 .
$$

Its regularity therefore depends on that of the function $x \rightarrow m\left(u\left(x, t^{*}\right)\right)$ which may be discontinuous at the shock point $x=x^{*}$. Thus $p$ is a decreasing function and has a $C^{1}$ regularity except at most at the shock point $x=x^{*}$ where the post- and preshock values 


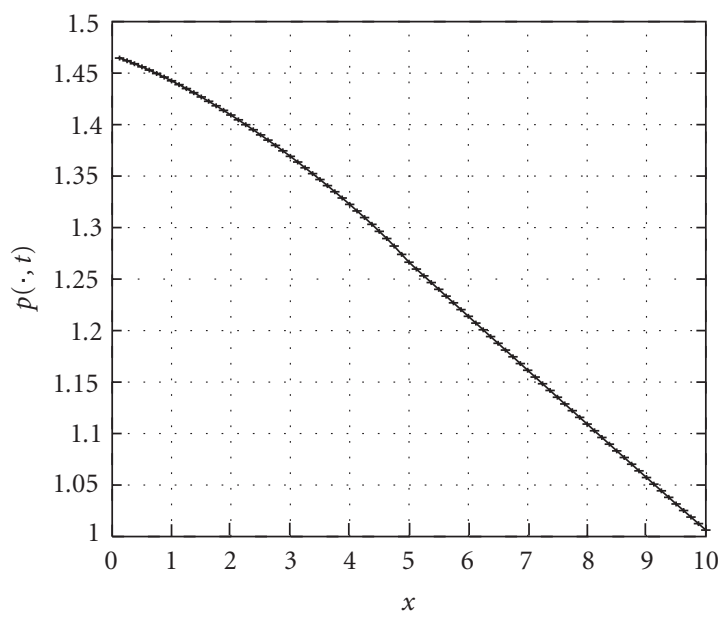

Figure 4.2. Graph of the pressure for $\lambda=4$.

of the derivative are given, respectively, by

$$
\begin{array}{r}
\frac{\partial p}{\partial x}\left(x_{+}^{*}, t^{*}\right)=\frac{-q}{m(0)}, \\
\frac{\partial p}{\partial x}\left(x_{-}^{*}, t^{*}\right)=\frac{-q}{m\left(u_{*}\right)},
\end{array}
$$

where $u_{*}$ is as defined by (4.17).

For example, for $\alpha=2$, we have

$$
u_{*}=\frac{1}{\sqrt{1+\lambda}}
$$

so that one has $m\left(u_{*}\right)=m(0)$ if $\lambda=3$. And for $\lambda$ greater (resp., less) than $3, m\left(u_{*}\right)$ is greater (resp., less) than $m(0)$. Thus, for $\lambda=3, p$ happens to be a smooth decreasing function in the $x$ variable. Figure 4.2 shows a typical profile for the pressure for some time $t>0$.

\section{The pseudo-2D case}

Consider now a rectangular domain $\Omega=(0, X) \times(0, Y)$ with

$$
\Gamma_{\text {in }}=\{0\} \times(0, Y), \quad \Gamma_{\text {out }}=\{X\} \times(0, Y),
$$

which comes to injecting water on the whole left boundary and recovering the oil on the whole right boundary. We call this configuration the pseudo-2D case because it can be assimilated to a one-dimensional flow as is stated in the following result. 
Theorem 5.1. Given $\lambda>0$ and $q>0$, let $u_{1}$ be the restriction to $(0, X)$ of the solution of the $1 D$ Riemann problem (3.1)-(3.2). Set, for $t>0$,

$$
p_{1}(x, y, t)=\int_{x}^{X} \frac{q}{m\left(u_{1}(s, t)\right)} d s, \quad(x, y) \in \Omega .
$$

Then $\left(u_{1}, p_{1}\right)$ is a solution of the system (1.1)-(1.5) in the sense of the definition (2.1) in the pseudo-2D case.

Proof. Let us first remark that, for $u=u_{1}, p_{1}$ defined by (5.2) is the unique solution of the problem (3.3). Indeed, for $\varphi \in \mathscr{V}$, we have

$$
\begin{aligned}
a\left(p_{1}, \varphi\right) & =\int_{\Omega} m\left(u_{1}\right) \nabla p_{1} \nabla \varphi d x d y \\
& =-\int_{\Omega} q \frac{\partial \varphi}{\partial x} d x d y=\int_{\Gamma_{\mathrm{in}}} q \varphi d y .
\end{aligned}
$$

Note also that as $u$ is the solution obtained in the previous section by solving the problem (4.3), we have, for all $t>0$,

$$
u(0, t)=1
$$

and as $q>0$ and $f$ is an increasing function, it suffices to impose a boundary condition for entering data only, that is, at $x=0$. Thus $v_{1}=u_{1}-1$, where $u_{1}$ is the restriction of $u$ on $(0, X)$, is the entropy solution of the initial boundary value problem

$$
(P) \begin{cases}\varepsilon u_{t}+q f(u+1)_{x}=0, & x \in(0, X), t \in(0, T), \\ u(0, t)=0, & t>0, \\ u(x, 0)=-1, & x \in(0, X) .\end{cases}
$$

Now it suffices to check that $\left(v_{1}, p_{1}\right)$ satisfies the inequality of the problem $\left(P_{u}\right)$. Let $k \in \mathbb{R}$ and $\phi \in D(\bar{\Omega} \times[0, T[), \phi \geq 0$. Define

$$
p_{k}(x)=\int_{x}^{X} \frac{q}{m(k+1)}=q(X-x) m(k+1)
$$

and define also the function

$$
\phi_{y}:(x, t) \longrightarrow \phi_{y}(x, t)=\phi(x, y, t)
$$

for all $y \in[0, Y], x \in[0, X]$, and $t \in\left[0, T\left[\right.\right.$. Such a function $\phi_{y}$ is in $D([0, X] \times[0, T[)$. Now as $v_{1}$ is an entropy solution of the problem (5.5), it satisfies the entropy inequality 
in the $1 \mathrm{D}$ case (cf., e.g., [6]), that is,

$$
\begin{aligned}
\int_{0}^{T} \int_{0}^{X}\{ & \left.\left|v_{1}-k\right| \frac{\partial \phi_{y}}{\partial t}+\operatorname{sg}\left(v_{1}-k\right)\left[q f\left(v_{1}+1\right)-q f(k+1)\right] \frac{\partial \phi_{y}}{\partial x}\right\} d x d t \\
& +\int_{0}^{T} \operatorname{sg}(k) q\left[\left(f\left(\gamma\left(v_{1}+1\right)\right)-f(k+1)\right) \phi_{y}\right]_{0}^{X} d t \\
& +\int_{0}^{X}\left|u_{0}-k\right| \phi_{y}(x, 0) d x \geq 0 .
\end{aligned}
$$

We now subtract the null quantity

$$
\int_{0}^{T} \int_{0}^{X} \operatorname{sg}\left(v_{1}-k\right) \frac{\partial}{\partial x}\left(-m(k+1) \frac{\partial p_{k}(x)}{\partial x}\right) f(k+1) \phi_{y} d x d t
$$

and then replace $q$ by $-m\left(v_{1}+1\right)\left(\partial p_{1} / \partial x\right)$ or by $-m(k+1)\left(\partial p_{k} / \partial x\right)$. Finally integrating in $y$ over $(0, Y)$, one obtains

$$
\begin{aligned}
\int_{Q}\left\{\mid v_{1}\right. & \left.-k \mid \phi_{t}+\operatorname{sg}\left(v_{1}-k\right)\left[h\left(v_{1}\right) \frac{\partial p_{1}}{\partial x}-h(k) \frac{\partial p_{k}}{\partial x} f(k)\right] \frac{\partial \phi}{\partial x}\right\} d x d y d t \\
& -\int_{Q} \operatorname{sg}\left(v_{1}-k\right) \operatorname{div}\left(h(k) \nabla p_{k}\right) \phi d x d y d t \\
& +\int_{0}^{T} \int_{0}^{Y} \operatorname{sg}(k)\left[\left(h\left(v_{1}\right) \frac{\partial p_{1}}{\partial x}-h(k) \frac{\partial p_{k}}{\partial x}\right) \phi\right]_{0}^{X} d y d t \\
& +\int_{\Omega}\left|u_{0}-k\right| \phi(x, y, 0) d x d y \geq 0 .
\end{aligned}
$$

Now it suffices to note that the third integral can be rewritten as

$$
\int_{0}^{T} \int_{\Gamma_{\text {in }} \cup \Gamma_{\text {out }}} \operatorname{sg}(k)\left(h\left(\gamma\left(v_{1}\right)\right) \nabla p_{1}-h(k) \nabla p_{k} \cdot \vec{n}\right) \phi d \sigma d t
$$

with $\vec{n}=(-1,0)$ on $\Gamma_{\text {in }}$ and $(1,0)$ on $\Gamma_{\text {out }}$. And as

$$
\nabla p_{1} \cdot \vec{n}=\nabla p_{k} \cdot \vec{n}=0
$$

on the rest of the boundary, the above integral can be rewritten on the whole boundary and the proof is done.

\section{Numerical simulations}

In this section, we present some numerical simulations concerning the $2 \mathrm{D}$ problem by using a nonconservative scheme of Harten type (cf. [1]) for the resolution of the hyperbolic problem

$$
u_{t}+\operatorname{div}(V f(u))=0, \quad(x, y) \in \Omega, t>0 .
$$


Table 6.1

\begin{tabular}{ccccc}
\hline$\mu_{w}$ & $\mu_{w}$ & $k$ & $\varepsilon$ & $q$ \\
\hline $1.10^{-3}$ & $4.10^{-3}$ & $2.10^{-13}$ & 0.225 & $3.510^{-7}$ \\
\hline
\end{tabular}

A better resolution of the shock, that is, of the oil-water interface is expected with this scheme because no interpolation is needed for the velocity terms as is the case in the conservative Harten scheme. Indeed, in the latter case, we need to have the values of the velocity terms at the points where the values of $u$ are calculated-for example, at the center of the grids-whereas they are computed at the interfaces of the grids. So some interpolation is necessary. The nonconservative scheme proposed calls for the values of the velocities $(V)$, of the saturation $(u)$, and of the pressure $(p)$ where they are calculated, that is, at the interfaces for $V$ and at the centre of the grids for $u$ and $p$.

Let $u_{i, j}^{n}$ be the approximation of $u$ at $(x=i \delta x, y=j \delta y, t=n \delta t)$. Define the difference operator $\Delta$ of type

$$
\Delta_{i+(1 / 2), j}(v)=v_{i+1, j}-v_{i, j},
$$

then the proposed scheme reads as follows:

$$
\begin{aligned}
u_{i, j}^{n+1}= & u_{i, j}^{n}+\frac{1}{2}\left(C_{i+(1 / 2), j}^{+} \Delta_{i+(1 / 2), j}\left(u^{n}\right)-C_{i-(1 / 2), j}^{-} \Delta_{i-(1 / 2), j}\left(u^{n}\right)\right) \\
& +\frac{1}{2}\left(C_{i, j+(1 / 2)}^{+} \Delta_{i, j+(1 / 2)}\left(u^{n}\right)-C_{i, j-(1 / 2)}^{-} \Delta_{i, j-(1 / 2)}\left(u^{n}\right)\right)
\end{aligned}
$$

with the functions $C$ defined as

$$
C_{i+(1 / 2), j}^{\bar{\mp}}=\frac{1}{2}\left[\left|\nu_{i+(1 / 2), j}+\gamma_{i+(1 / 2), j}\right| \pm\left(\nu_{i+(1 / 2), j}+\gamma_{i+(1 / 2), j}\right)\right] .
$$

It is formulated just as the second-order Harten scheme (cf. [18]) but with the functions $\nu$ and $\gamma$ defined differently. In fact, here we have

$$
v_{i+(1 / 2), j}=V_{x, i+(1 / 2), j} \lambda_{x} \frac{\Delta_{i+(1 / 2), j}(f(u))}{\Delta_{i+(1 / 2), j} u}
$$

with $V_{x}$ the horizontal component of $V$ and $\lambda_{x}=\delta t / \delta x$. The same type of modification applies to the second-order terms $\gamma$. One can refer to [1] for the details concerning the derivation of the above scheme and some properties in view of its convergence.

Figure 6.1 shows the profile of the saturation obtained in the pseudo-2D case for the standard set of data given in Table 6.1.

We notice the good resolution for the interface and the fact that the flow is independent of the $y$ - variable so that it can be assimilated to a $1 \mathrm{D}$ flow. For this sake, 


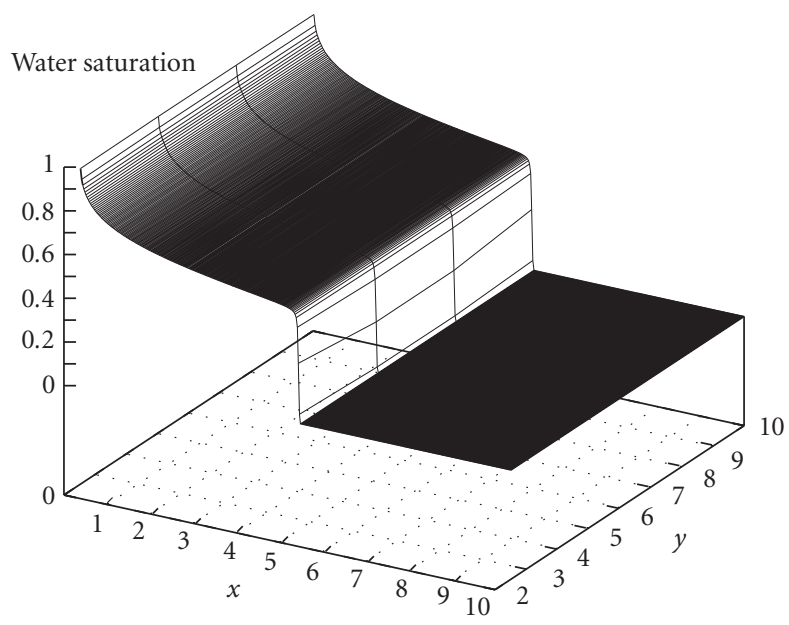

Figure 6.1. A 2D plot of the water saturation computed with the 2D scheme.

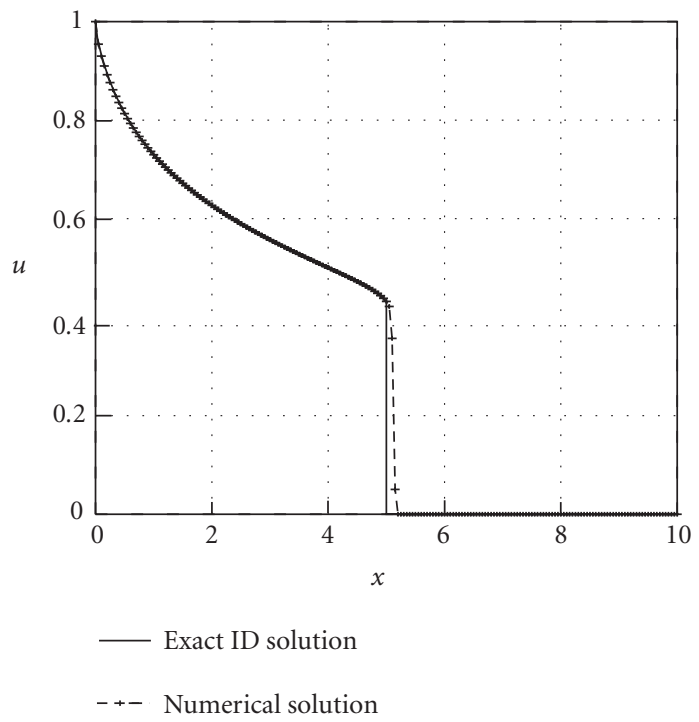

Figure 6.2. Comparison of the 2D numerical and the exact 1D profile.

in Figure 6.2, we compare the saturation profile computed for any given $y$ with the exact $1 \mathrm{D}$ solution obtained by the resolution of the corresponding Riemann problem. The results are in good agreement.

Figure 6.3 shows the profile of the oil-water interface in time. The observed linear profile enables us to compute the constant numerical speed which is given by

$$
c_{\text {num }}=\text { slope } \times t_{\text {ref }}=0.33 \times 7.12 \times 10^{-6}=2.5 \times 10^{-6} \mathrm{~m} / \mathrm{s}
$$




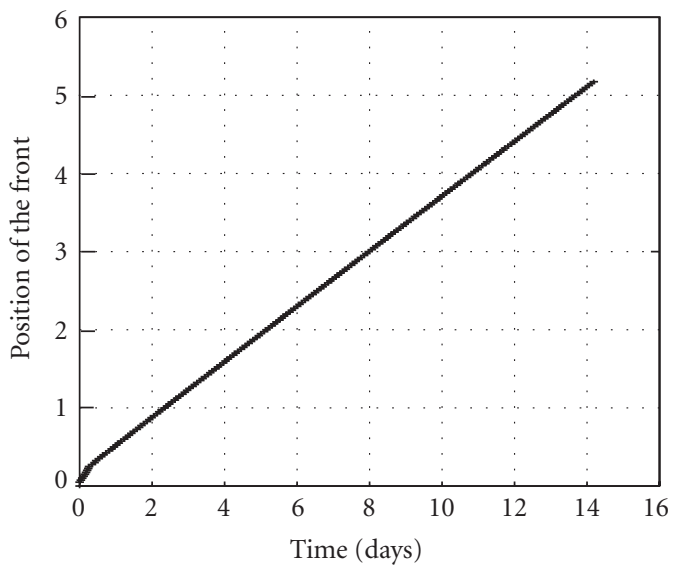

Figure 6.3. Position of the oil-water interface in time.

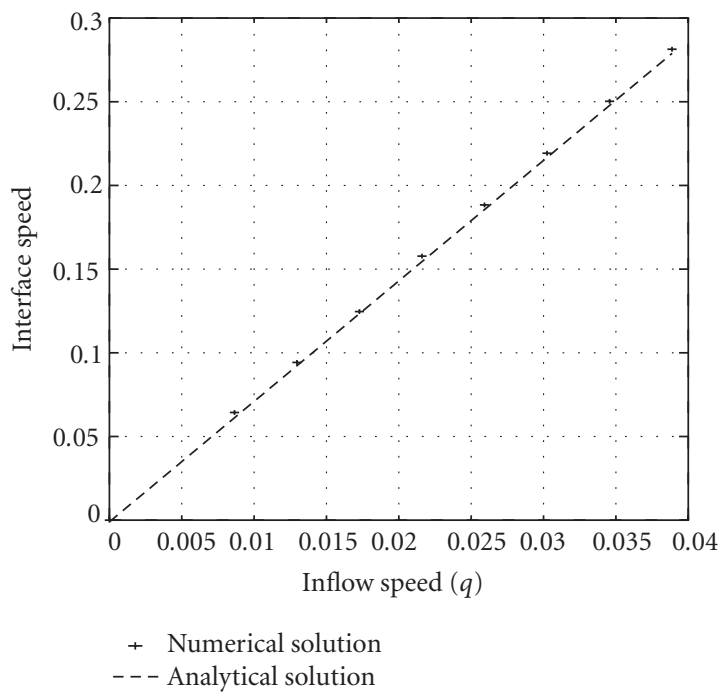

Figure 6.4. The interface speed with respect to the inflow speed $q$.

whereas the analytical value, obtained by (4.19), is

$$
c=\frac{q}{\varepsilon} \frac{1+\sqrt{1+\lambda}}{2}=2,51 \times 10^{-6} \mathrm{~m} / \mathrm{s}
$$

Other comparisons can be done to show the concordance of the numerical results obtained with the analytical ones. We end up with two profiles for the interface speed 


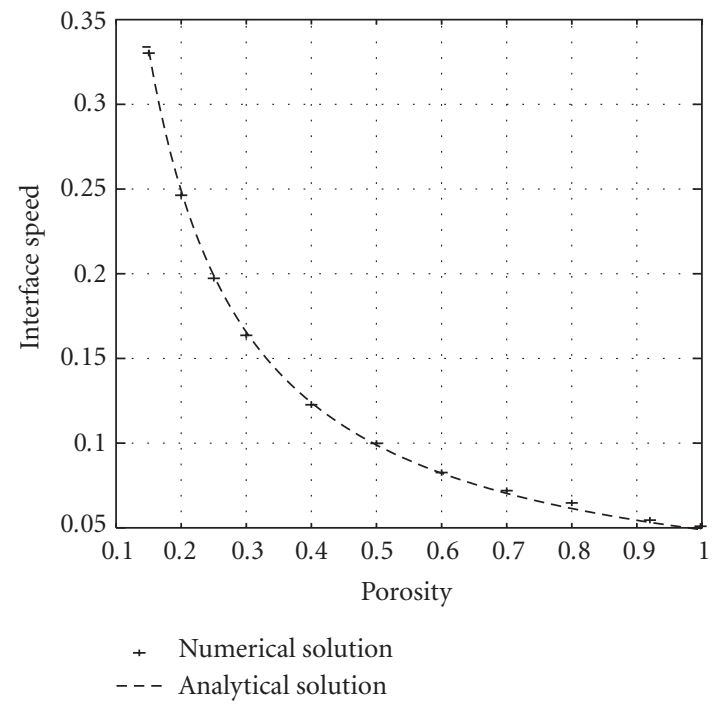

Figure 6.5. The interface speed as a function of the porosity for $\lambda=4$.

with respect to the inflow speed $q$ (Figure 6.4) and the porosity $\varepsilon$ (Figure 6.5). In the two cases, the computed values coincide with those expected according to the formula (4.19).

\section{References}

[1] A. Abbassi, G. Namah, and M. Saad, A nonconservative numerical scheme for flow computations in porous media, preprint.

[2] M. B. Allen, Numerical modelling of multiphase flow in porous media, Adv. Water Resources 8 (1985), 162-187.

[3] R. J. Atkin and R. E. Craine, Continuum theories of mixtures: basic theory and historical development, Quart. J. Mech. Appl. Math. 29 (1976), no. 2, 209-244.

[4] K. Aziz and A. Settari, Petrolum Reservoir Simulation, Elsevier, New York, 1990.

[5] C. Bardos, A. Y. LeRoux, and J.-C. Nédélec, First order quasilinear equations with boundary conditions, Comm. Partial Differential Equations 4 (1979), no. 9, 1017-1034.

[6] R. M. Bowen, Compressible porous media models by the use of the theory of mixtures, Quarterly Journal of Applied Maths. 20 (1982), no. 6, 697-735.

[7] S. E. Buckley and M. C. Leverett, Mechanism of fluid displacement in sands, Transactions, American Institute of Mining, Metallurgical and Petroleum Engineers 146 (1942), 107-116.

[8] G. Chavent and J. Jaffre, Mathematical Models and Finite Elements for Reservoir Simulation, Studies in Mathematics and Its Applications, vol. 17, North-Holland, Amsterdam, 1986.

[9] H. Frid, Solution to the initial-boundary-value problem for the regularized Buckley-Leverett system, Acta Appl. Math. 38 (1995), no. 3, 239-265.

[10] G. Gagneux and M. Madaune-Tort, Analyse mathématique de modèles non linéaires de l'ingénierie pétrolière, Mathématiques et Applications, vol. 22, Springer, Paris, 1995.

[11] E. Godlewski and P.-A. Raviart, Hyperbolic Systems of Conservation Laws, Mathématiques \& Applications, vol. 3/4, Ellipses, Paris, 1991. 
[12] A. Harten, High resolution schemes for hyperbolic conservation laws, J. Comput. Phys. 9 (1983), no. 3, 357-393.

[13] A. Y. LeRoux, Approximation de quelques problèmes hyperboliques non linéaires, Thèse, Université de Rennes, Rennes, 1979.

[14] K. R. Rajagopal and L. Tao, Mechanics of Mixtures, Series on Advances in Mathematics for Applied Sciences, vol. 35, World Scientific, New Jersey, 1995.

[15] H. J. Schroll and A. Tveito, Local existence and stability for a hyperbolic-elliptic system modeling two-phase reservoir flow, Electron. J. Differential Equations 2000 (2000), no. 4, 1-28.

[16] C. Truesdell, Rational mechanics of deformation and flow. II: a general theory of constitutive equations, Bul. Inst. Politehn. Iaşi (N.S.) 14(18) (1968), no. 1/2, 131-136.

Adil Abbassi: Laboratoire Mathématiques de Besançon, UMR 6623 CNRS, Université de Franche-Comté, 16 route de Gray, 25030 Besançon Cedex, France

E-mail address: abbassi@math.univ-fcomte.fr

Gawtum Namah: Laboratoire Mathématiques de Besançon, UMR 6623 CNRS, Université de Franche-Comté, 16 route de Gray, 25030 Besançon Cedex, France

E-mail address: gawtum.namah@math.univ-fcomte.fr 


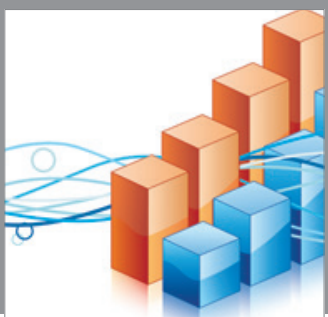

Advances in

Operations Research

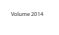

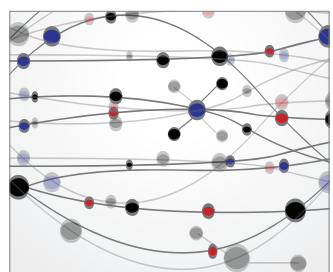

\section{The Scientific} World Journal
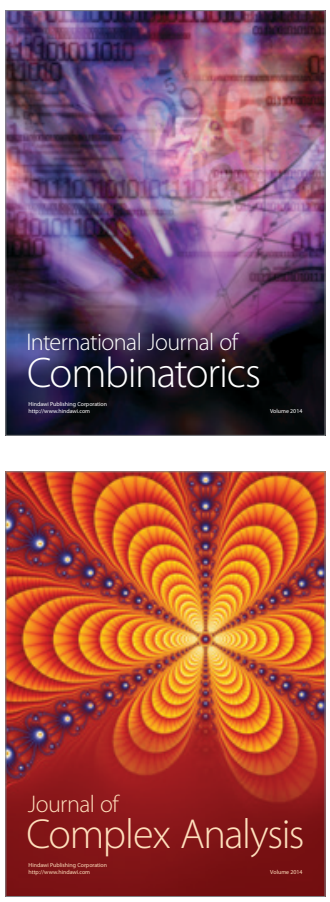

International Journal of

Mathematics and

Mathematical

Sciences
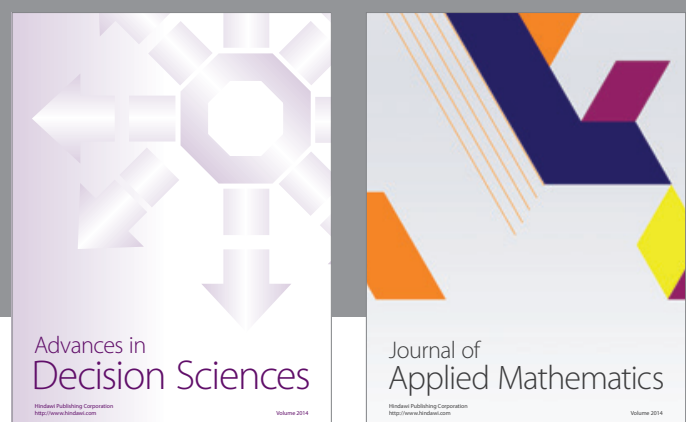

Journal of

Applied Mathematics
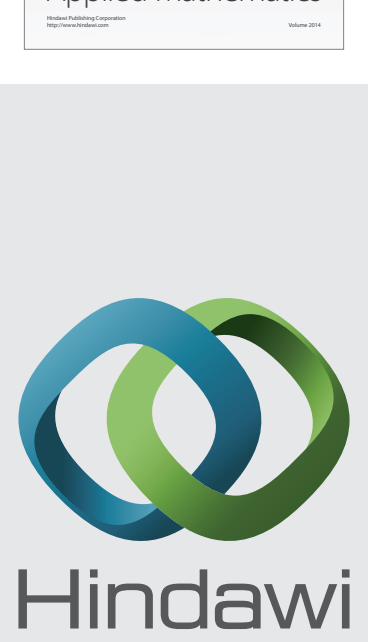

Submit your manuscripts at http://www.hindawi.com
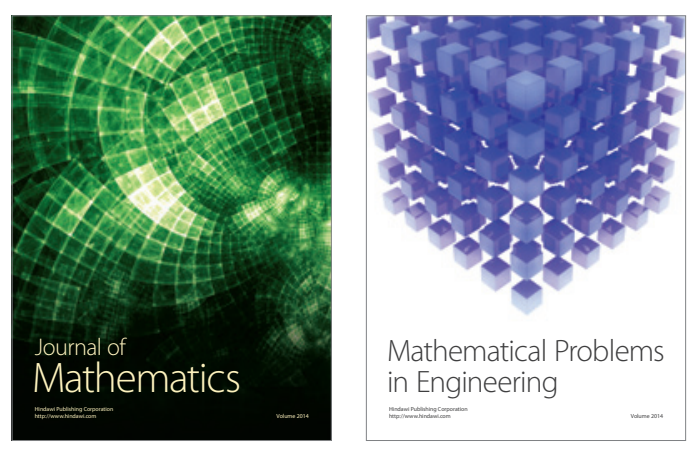

Mathematical Problems in Engineering
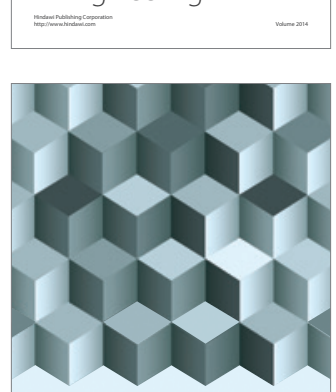

Journal of

Function Spaces
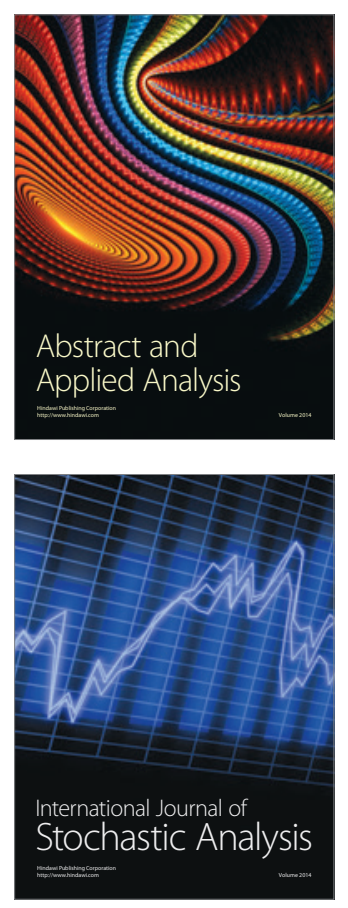

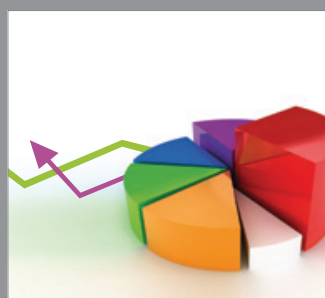

ournal of

Probability and Statistics

Promensencen
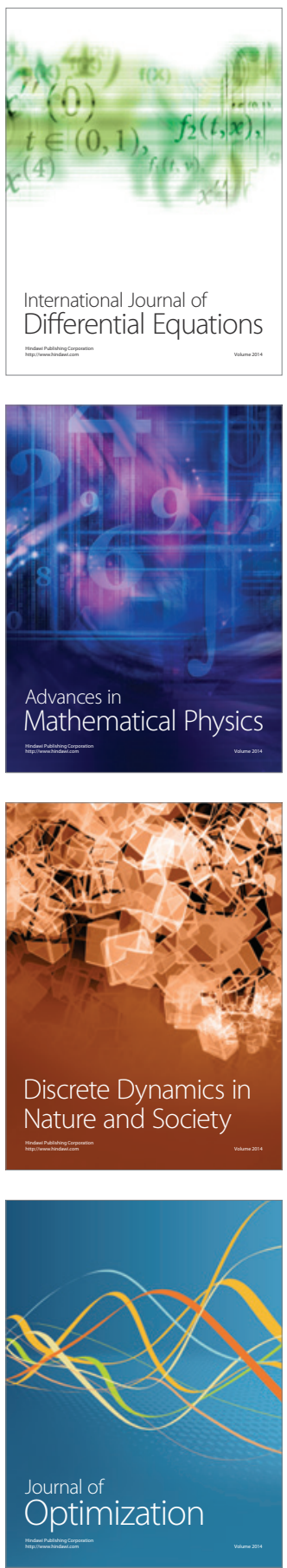